\title{
Method of External Potential in Solution of Cauchy Mixed Problem for the Heat Equation
}

\author{
T. Sh. Kalmenov and N. E. Tokmagambetov \\ Institute of Mathematics and Mathematical Modeling, MES RK, Shevchenko Street, 28, Almaty 050010, Kazakhstan \\ Correspondence should be addressed to N. E. Tokmagambetov; tokmagam@list.ru
}

Received 30 May 2013; Accepted 18 July 2013

Academic Editors: S. Filippas and K. Lurie

Copyright (C) 2013 T. Sh. Kalmenov and N. E. Tokmagambetov. This is an open access article distributed under the Creative Commons Attribution License, which permits unrestricted use, distribution, and reproduction in any medium, provided the original work is properly cited.

\begin{abstract}
Numerous research works are devoted to study Cauchy mixed problem for model heat equations because of its theoretical and practical importance. Among them we can notice monographers Vladimirov (1988), Ladyzhenskaya (1973), and Tikhonov and Samarskyi (1980) which demonstrate main research methods, such as Fourier method, integral equations method, and the method of a priori estimates. But at the same time to represent the solution of Cauchy mixed problem in integral form by given and known functions has not been achieved up to now. This paper completes this omission for the one-dimensional heat equation.
\end{abstract}

\section{Introduction}

Partial differential equations of parabolic type are widely represented in the study of heat conductivity and diffusion process. Numerous research works are devoted to study Cauchy mixed problem for model heat equations because of its theoretical and practical importance. Among them we can notice monographers [1-3] which demonstrate main research methods, such as Fourier method, integral equations method, and method of a priori estimates. But at the same time to represent the solution of Cauchy mixed problem in integral form by given and known functions has not been achieved up to now. This paper completes this omission for the onedimensional heat equation.

Exterior potential method as a special continuation of a solution for all half-space is widely used under the solution of Cauchy mixed problem. Our idea is based on a representation possibility of general solution only in the form of volume potential excluding surface integrals. Thus the system of integral equations obtained by this method allows us to construct the solution in quadrature.

\section{Material and Methods}

Consider the following problem in a plane domain $\Omega=\{0<$ $x<1,0<t<T\}$.
Cauchy Mixed Problem. To find a regular solution of the following equation in $\Omega$

$$
\nabla u(x, t) \equiv\left(\frac{\partial}{\partial t}-\frac{\partial^{2}}{\partial x^{2}}\right) u(x, t)=f(x, t),
$$

with the initial condition

$$
u(x, 0)=0
$$

and boundary conditions

$$
u(0, t)=u(1, t)=0 .
$$

Our goal is to construct a classical solution of the problem (1)-(3) in a quadrature. We will seek a solution in the form of sum of three volume potentials:

$$
u(x, t)=u_{f}(x, t)+u_{g_{0}}(x, t)+u_{g_{1}}(x, t),
$$

where

$$
\begin{aligned}
u_{f}(x, t) & =\left(\varepsilon_{1} * f\right)(x, t) \\
& =\int_{0}^{t} d \tau \int_{0}^{1} \frac{\exp \left(-(x-\xi)^{2} / 4(t-\tau)\right)}{2 \sqrt{\pi(t-\tau)}} f(\xi, \tau) d \xi,
\end{aligned}
$$




$$
\begin{aligned}
u_{g_{0}}(x, t)= & \left(\varepsilon_{1} * g_{0}\right)(x, t) \\
= & \int_{0}^{t} d \tau \int_{-\infty}^{0} \frac{\exp \left(-(x-\xi)^{2} / 4(t-\tau)\right)}{2 \sqrt{\pi(t-\tau)}} \\
& \times g_{0}(\xi, \tau) d \xi \\
u_{g_{1}}(x, t)= & \left(\varepsilon_{1} * g_{1}\right)(x, t) \\
= & \int_{0}^{t} d \tau \int_{1}^{+\infty} \frac{\exp \left(-(x-\xi)^{2} / 4(t-\tau)\right)}{2 \sqrt{\pi(t-\tau)}} g_{1}(\xi, \tau) d \xi .
\end{aligned}
$$

Here $\varepsilon_{1}(x, t)=\theta(t)(2 \sqrt{\pi t})^{-1} \exp \left(-|x|^{2} / 4 t\right)$ is a fundamental solution of the heat equation (1) and

$$
\theta(t)= \begin{cases}1, & t>0 \\ 0, & t \leq 0\end{cases}
$$

is Heaviside theta-function.

It should be noticed that the heat potential $u_{f}(x, t)$ satisfies the following boundary condition:

$$
\begin{aligned}
& -\frac{u(x, t)}{2} \\
& +\int_{0}^{t}\left[\frac{(x-\xi)}{4 \sqrt{\pi}(t-\tau)}\right. \\
& \times \exp \left(-\frac{(x-\xi)^{-1}}{4(t-\tau)}\right) u(\xi, \tau) \\
& -\frac{1}{2 \sqrt{\pi(t-\tau)}} \\
& \left.\times\left.\exp \left(-\frac{(x-\xi)^{-1}}{4(t-\tau)}\right) \frac{\partial u(\xi, \tau)}{\partial \xi}\right|_{\xi=0} ^{\xi=1}\right] d \tau=0 \\
& \forall(x, t) \in \partial \Omega \text {, }
\end{aligned}
$$

where $\partial \Omega$ is a boundary of the domain $\Omega$. Note that in works $[4,5]$ differential operators with nonlocal boundary conditions are investigated as above.

It is easy to verify that the first term in representation (4), $u_{f}(x, t)$, is a solution of nonhomogeneous equation (1) and the second and third ones, $u_{g_{0}}(x, t), u_{g_{1}}(x, t)$, are solutions of homogeneous equation. Consequently, representation (4) gives a solution of non-homogeneous equation (1) satisfying initial condition (2) for arbitrary $g_{0}(x, t), g_{1}(x, t)$.

Our aim is to choose unknown functions $g_{0}(x, t)$ and $g_{1}(x, t)$ such that the solution will satisfy boundary condition (3).

We will seek functions $g_{0}(x, t), g_{1}(x, t)$ in the forms

$$
\begin{gathered}
g_{0}(x, t)=x \theta(-x) g_{0}(t), \\
g_{1}(x, t)=(x-1) \theta(x-1) g_{1}(t) .
\end{gathered}
$$

Let $\varphi(x)$ be periodical function with period $2 \pi$ defined on the interval $(0,2 \pi)$ by the formula $\varphi(x)=(x / 2)-\left(x^{2} / 4 \pi\right)$. This function can be represented by the formula $\varphi(x)=(\pi / 6)-$ $(1 / 2 \pi) \sum_{k=-\infty}^{k=+\infty}\left(1 / k^{2}\right) \exp (i k x)$ out of the interval $[0,2 \pi]$.

We introduce the following notations:

$$
\begin{gathered}
G_{0}(t)=\int_{-\infty}^{+\infty}\left(\varphi_{0}(x)+\frac{x^{2}}{4 \pi}\right) \frac{d^{2}}{d x^{2}} \exp \left(-\frac{|x|^{2}}{4(\pi)^{2} t}\right) d x \\
G_{1}(t)=\int_{-\infty}^{+\infty}\left(\varphi_{0}(x-\pi)+\frac{(x-\pi)^{2}}{4 \pi}\right) \frac{d^{2}}{d x^{2}} \\
\times \exp \left(-\frac{|x|^{2}}{4(\pi)^{2} t}\right) d x \\
F_{0}(t)=\left.u_{f}(x, t)\right|_{x=0} \\
=\int_{0}^{t} d \tau \int_{0}^{1} \frac{\exp \left(-|\xi|^{2} / 4(t-\tau)\right)}{2 \sqrt{\pi(t-\tau)}} \\
F_{1}(t)=\left.u_{f}(x, t)\right|_{x=1} \\
=\int_{0}^{t} d \tau \int_{0}^{1} \frac{\exp \left(-(1-\xi)^{2} / 4(t-\tau)\right)}{2 \sqrt{\pi(t-\tau)}} \\
\times f(\xi, \tau) d \xi .
\end{gathered}
$$

\section{Results and Discussion}

The main result of this paper is as follows.

Theorem 1. Let $f(x, t) \in C^{\alpha, \alpha / 2}(\bar{\Omega}), 0<\alpha<1$, and then the solution of the problem (1)-(3) is represented by formula (4), where $g_{0}(x, t)$ and $g_{1}(x, t)$ are given by formulae $(8)$ :

$$
\begin{gathered}
g_{0}(t)=\frac{d^{2}}{d t^{2}} \int_{0}^{t}\left[G_{0}(t-\tau) F_{0}(\tau)-G_{1}(t-\tau) F_{1}(\tau)\right] d \tau, \\
g_{1}(t)=-\frac{d^{2}}{d t^{2}} \int_{0}^{t}\left[G_{0}(t-\tau) F_{1}(\tau)-G_{1}(t-\tau) F_{0}(\tau)\right] d \tau .
\end{gathered}
$$

To prove the theorem, the main role plays the following.

Lemma 2. Let unknown functions be given by the formulae (8). Then $g_{0}(t)$ and $g_{1}(t)$ satisfy the system of equations

$$
\begin{aligned}
& \int_{0}^{t} \frac{g_{0}(\tau)}{2 \sqrt{\pi(t-\tau)}} d \tau \\
& \quad-\int_{0}^{t} \frac{\exp (-1 /(4 \pi(t-\tau)))}{2 \sqrt{\pi(t-\tau)}} g_{1}(\tau) d \tau=\frac{d}{d t} F_{0}(t),
\end{aligned}
$$




$$
\begin{aligned}
& \int_{0}^{t} \frac{g_{1}(\tau)}{2 \sqrt{\pi(t-\tau)}} d \tau \\
& \quad-\int_{0}^{t} \frac{\exp (-1 /(4 \pi(t-\tau)))}{2 \sqrt{\pi(t-\tau)}} g_{0}(\tau) d \tau=-\frac{d}{d t} F_{1}(t),
\end{aligned}
$$

where $F_{0}(t), F_{1}(t)$ are defined by formula (10).

Proof. By substituting function (4) in boundary condition (3) and taking account of (10), we get a system of interval equations with respect to unknown functions $g_{0}(t)$ and $g_{1}(t)$ :

$$
\begin{gathered}
\int_{0}^{t} g_{0}(\tau) d \tau \int_{-\infty}^{0} \frac{\exp \left(-\xi^{2} / 4 \pi(t-\tau)\right)}{2 \sqrt{\pi(t-\tau)}} \xi d \xi \\
\quad+\int_{0}^{t} g_{1}(\tau) d \tau \int_{1}^{+\infty} \frac{\exp \left(-\xi^{2} / 4 \pi(t-\tau)\right)}{2 \sqrt{\pi(t-\tau)}}(\xi-1) d \xi \\
\quad=-F_{0}(t), \\
\int_{0}^{t} g_{0}(\tau) d \tau \int_{-\infty}^{0} \frac{\exp \left(-(\xi-1)^{2} / 4 \pi(t-\tau)\right)}{2 \sqrt{\pi(t-\tau)}} \xi d \xi \\
+\int_{0}^{t} g_{1}(\tau) d \tau \int_{1}^{+\infty} \frac{\exp \left(-(\xi-1)^{2} / 4 \pi(t-\tau)\right)}{2 \sqrt{\pi(t-\tau)}} \\
\times(\xi-1) d \xi=-F_{1}(t) .
\end{gathered}
$$

Integrating by part it is easy to verify correctness of the following equations:

$$
\begin{aligned}
& \int_{-\infty}^{0} \frac{\exp \left(-\xi^{2} / 4 \pi(t-\tau)\right)}{2 \sqrt{\pi(t-\tau)}} \xi d \xi \\
&=-\frac{2}{\sqrt{\pi(t-\tau)}} \int_{-\infty}^{0} \frac{\partial}{\partial \xi} \exp \left(-\frac{\xi^{2}}{4 \pi(t-\tau)}\right) d \xi \\
&=-\frac{\sqrt{(t-\tau)}}{\sqrt{\pi}} \\
& \int_{1}^{\infty} \frac{\exp \left(-\xi^{2} / 4 \pi(t-\tau)\right)}{2 \sqrt{\pi(t-\tau)}}(\xi-1) d \xi \\
& \quad=\frac{\sqrt{(t-\tau)}}{\sqrt{\pi}}-\frac{1}{\sqrt{\pi}} \int_{1 / 2 \sqrt{(t-\tau)}}^{\infty} \exp \left(-\xi^{2}\right) d \xi .
\end{aligned}
$$

After putting $\xi:=1-\xi$ in integrals (13), our system can be transformed to

$$
\begin{aligned}
& \int_{0}^{t} \frac{\sqrt{(t-\tau)}}{\sqrt{\pi}} g_{0}(\tau) d \tau \\
& -\int_{0}^{t}\left[\frac{\sqrt{(t-\tau)}}{\sqrt{\pi}} \exp \left(-\frac{1}{4 \sqrt{(t-\tau)}}\right)\right. \\
& \left.\quad-\frac{1}{\sqrt{\pi}} \int_{1 / 2 \sqrt{(t-\tau)}}^{\infty} \exp \left(-\xi^{2}\right) d \xi\right] \\
& \quad \times g_{1}(\tau) d \tau=F_{0}(t), \\
& \int_{0}^{t}\left[\frac{\sqrt{(t-\tau)}}{\sqrt{\pi}} \exp \left(-\frac{1}{4 \sqrt{(t-\tau)}}\right)\right. \\
& \left.-\frac{1}{\sqrt{\pi}} \int_{1 / 2 \sqrt{(t-\tau)}}^{\infty} \exp \left(-\xi^{2}\right) d \xi\right] \\
& \times g_{0}(\tau) d \tau-\int_{0}^{t} \frac{\sqrt{(t-\tau)}}{\sqrt{\pi}} g_{1}(\tau) d \tau=F_{1}(t) .
\end{aligned}
$$

By differentiating these relations we obtain the system (12). The lemma is proved.

Now we will construct the solution of the system (12) by using the Laplace transformation properties:

$$
\begin{gathered}
F\left(\frac{\exp \left(-\alpha^{2} / 4 t\right)}{\sqrt{t}}\right)=\sqrt{\pi} \frac{\exp (\alpha \sqrt{p})}{\sqrt{p}}, \\
F(f * g)=\tilde{f}(p) \tilde{g}(p), \\
F\left(\frac{d}{d t} f\right)=p \tilde{f}(p) .
\end{gathered}
$$

The system (12) can be transformed in the next form:

$$
\begin{gathered}
\frac{\widetilde{g}_{0}(p)}{\sqrt{p}}-\frac{\widetilde{g}_{1}(p)}{\sqrt{p}} \exp (-\sqrt{p})=p \widetilde{F}_{0}(p), \\
-\frac{\widetilde{g}_{0}(p)}{\sqrt{p}} \exp (-\sqrt{p})+\frac{\widetilde{g}_{1}(p)}{\sqrt{p}}=-p \widetilde{F}_{1}(p),
\end{gathered}
$$

where $\widetilde{g}_{0}(p)=F\left(g_{0}\right)(p), \widetilde{g}_{1}(p)=F\left(g_{1}\right)(p)$ are images of the Laplace transform. After solving this system with respect to $\widetilde{g}_{0}(p)$ and $\widetilde{g}_{1}(p)$ we find

$$
\begin{gathered}
\widetilde{g}_{0}(p)=\frac{p^{2}}{\sqrt{p}}\left(\frac{\widetilde{F}_{0}(p)-\widetilde{F}_{1}(p) \exp (-\sqrt{p})}{1-\exp (-2 \sqrt{p})}\right), \\
\widetilde{g}_{1}(p)=-\frac{p^{2}}{\sqrt{p}}\left(\frac{\widetilde{F}_{1}(p)-\widetilde{F}_{0}(p) \exp (-\sqrt{p})}{1-\exp (-2 \sqrt{p})}\right) .
\end{gathered}
$$


Since Laplace inverse transformation of function $(1 / \sqrt{p})(1 /(1-\exp (-2 \sqrt{p})))$ has no table form, so by using the expansions

$$
\begin{gathered}
\frac{1}{\sqrt{p}} \frac{1}{1-\exp (-2 \sqrt{p})}=\sum_{k=0}^{\infty} \frac{1}{\sqrt{p}} \exp (-2 \sqrt{p} k), \\
\frac{1}{\sqrt{p}} \frac{\exp (-\sqrt{p})}{1-\exp (-2 \sqrt{p})}=\sum_{k=0}^{\infty} \frac{1}{\sqrt{p}} \exp (-(2 k+1) \sqrt{p})
\end{gathered}
$$

and table values of the Laplace inverse transformation,

$$
\begin{gathered}
F^{-1}\left(\frac{\exp (-2 k \sqrt{p})}{\sqrt{p}}\right)=\frac{1}{\sqrt{\pi}} \frac{\exp \left(-(2 k)^{2} / 4 t\right)}{\sqrt{t}}, \\
F^{-1}\left(\frac{\exp (-(2 k+1) \sqrt{p})}{\sqrt{p}}\right)=\frac{1}{\sqrt{\pi}} \frac{\exp \left(-(2 k+1)^{2} / 4 t\right)}{\sqrt{t}} .
\end{gathered}
$$

Then from (18) we obtain

$$
\begin{gathered}
g_{0}(t)=\frac{d^{2}}{d t^{2}} \int_{0}^{t}\left[G_{0}(t-\tau) F_{0}(\tau)-G_{1}(t-\tau) F_{1}(\tau)\right] d \tau, \\
g_{1}(t)=-\frac{d^{2}}{d t^{2}} \int_{0}^{t}\left[G_{0}(t-\tau) F_{1}(\tau)-G_{1}(t-\tau) F_{0}(\tau)\right] d \tau,
\end{gathered}
$$

where

$$
\begin{aligned}
G_{0}(t) & =F^{-1}\left(\sum_{k=0}^{\infty} \frac{\exp (-2 k \sqrt{p})}{\sqrt{p}}\right) \\
& =\sum_{k=0}^{\infty}\left(F^{-1}\left(\frac{\exp (-2 k \sqrt{p})}{\sqrt{p}}\right)\right) \\
& =\frac{1}{\sqrt{\pi}} \sum_{k=0}^{\infty} \frac{\exp \left(-(2 k)^{2} / 4 t\right)}{\sqrt{t}}, \\
G_{1}(t) & =F^{-1}\left(\sum_{k=0}^{\infty} \frac{\exp (-(2 k+1) \sqrt{p})}{\sqrt{p}}\right) \\
& =\frac{1}{\sqrt{\pi}} \sum_{k=0}^{\infty} \frac{\exp \left(-(2 k+1)^{2} / 4 t\right)}{\sqrt{t}} .
\end{aligned}
$$

To complete the proof of the theorem we represent functions $G_{0}(t)$ and $G_{1}(t)$ by integrals of known functions.

As long as $\delta(x-2 k \pi),(k=0, \pm 1, \pm 2, \ldots)$ is a tempered distribution [6, page 112], and then we have

$$
\left(\delta(x-2 k \pi), \exp \left(-\frac{x^{2}}{4 \pi^{2} t}\right)\right)=\exp \left(-\frac{(2 k)^{2}}{4 t}\right)
$$

Therefore, the following relations hold:

$$
\begin{gathered}
\sum_{k=-\infty}^{\infty}\left(\delta(x-2 k \pi), \exp \left(-\frac{x^{2}}{4 \pi^{2} t}\right)\right)=\sum_{k=-\infty}^{\infty} \exp \left(-\frac{(2 k)^{2}}{4 t}\right) \\
\sum_{k=-\infty}^{\infty}\left(\delta(x-(2 k+1) \pi), \exp \left(-\frac{x^{2}}{4 \pi^{2} t}\right)\right) \\
=\sum_{k=-\infty}^{\infty} \exp \left(-\frac{(2 k+1)^{2}}{4 t}\right) .
\end{gathered}
$$

We define function $\varphi_{0}(x)=(x / 2)-\left(x^{2} / 4 \pi\right)$ on the interval $[0,2 \pi]$ and then continue it periodically on all axes $(-\infty, \infty)$. It is shown in $[6$, page 113$]$

$$
\sum_{k=-\infty}^{\infty} \delta(x-2 k \pi)=\frac{d^{2}}{d x^{2}}\left(\varphi_{0}(x)+\frac{x^{2}}{4 \pi}\right),
$$

where $\left(d^{2} / d x^{2}\right)$ is regarded in the general function sense.

From here and relation (25) we conclude that

$$
\begin{aligned}
\sum_{k=0}^{\infty} \exp \left(-\frac{(2 k)^{2}}{4 t}\right) \\
=\frac{1}{2}\left(\frac{d^{2}}{d x^{2}}\left(\varphi_{0}(x)+\frac{x^{2}}{4 \pi}\right), \exp \left(-\frac{x^{2}}{4 \pi^{2} t}\right)\right) \\
=\frac{1}{2} \int_{-\infty}^{\infty}\left(\varphi_{0}(x)+\frac{x^{2}}{4 \pi}\right) \frac{d^{2}}{d x^{2}} \exp \left(-\frac{x^{2}}{4 \pi^{2} t}\right) d x .
\end{aligned}
$$

Analogically, the relation

$$
\begin{aligned}
& \sum_{k=0}^{\infty} \exp \left(-\frac{(2 k+1)^{2}}{4 t}\right) \\
&=\frac{1}{2} \int_{-\infty}^{\infty}\left(\varphi_{0}(x-\pi)+\frac{(x-\pi)^{2}}{4 \pi}\right) \frac{d^{2}}{d x^{2}} \\
& \times \exp \left(-\frac{x^{2}}{4 \pi^{2} t}\right) d x
\end{aligned}
$$

follows from (26).

We obtain formula (9) after substituting the last relations in (22) and (23).

From (23) we have $G_{1}(t) \in C^{\infty}[0,1]$, and from (22) $\left(G_{0}(t)-(1 / \sqrt{\pi} t)\right) \in C^{\infty}[0,1]$.

Since $f(x, t) \in C^{1+\alpha, 1+(\alpha / 2)}(\bar{\Omega}), 0<\alpha<1$, then $F_{0}(t), F_{1}(t) \in C^{1+(\alpha / 2)}(0, T)$. Therefore it is not difficult to establish $g_{0}(t), g_{1}(t) \in C^{1+(\alpha / 2)}[0, T]$.

Consequently, representation of the solution $u(x, t) \in$ $C^{2+\alpha, 1+(\alpha / 2)}(\bar{\Omega})$ in the form (4) is established. The theorem is fully proved. 


\section{References}

[1] V. S. Vladimirov, Equations of Mathematical Physics, "Nauka", Moscow, Russia, 1988.

[2] O. A. Ladyzhenskaya, Boundary Value Problems for the Parabolic Equations, Nauka, 1973.

[3] A. N. Tikhonov and A. A. Samarskyi, Equations of Mathematical Physics, Nauka, 1980.

[4] T. Sh. Kal'menov and N. E. Tokmagambetov, "On a boundary condition for a spatially one-dimensional generalized volume heat potential," Izvestiya Natsional noĭ Akademii Nauk Respubliki Kazakhstan, no. 5, pp. 3-9, 2010.

[5] D. Suragan and N. Tokmagambetov, "On transparent boundary conditions for the high-order heat equation," Siberian Electronic Mathematical Reports, vol. 10, pp. 141-149, 2013.

[6] V. A. Ditkin and A. P. Prudnikov, Integral Transformations and Operator Calculus, Nauka, 1984. 


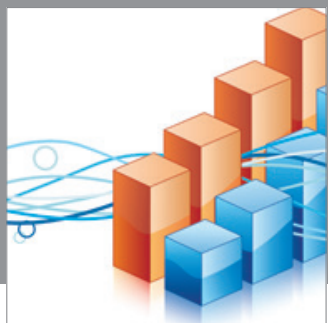

Advances in

Operations Research

mansans

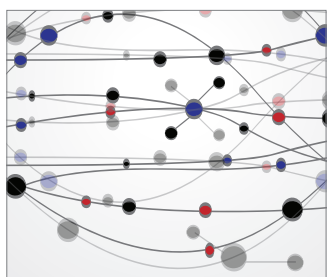

The Scientific World Journal
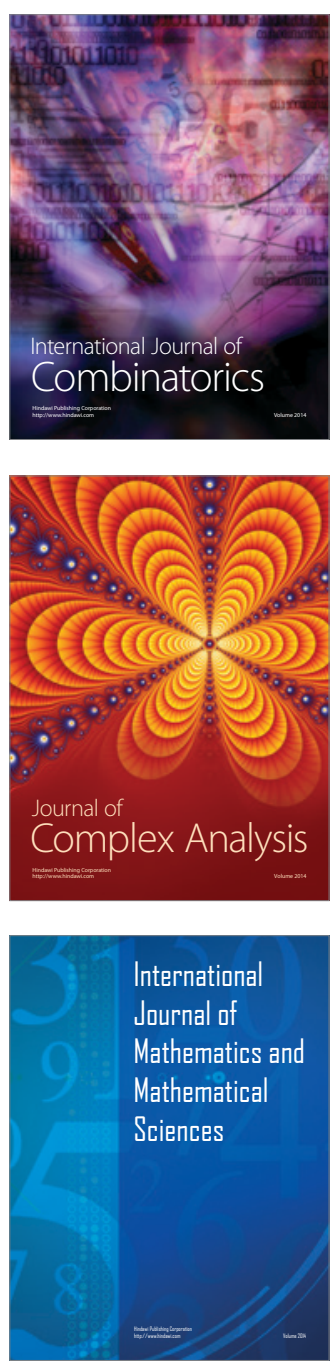
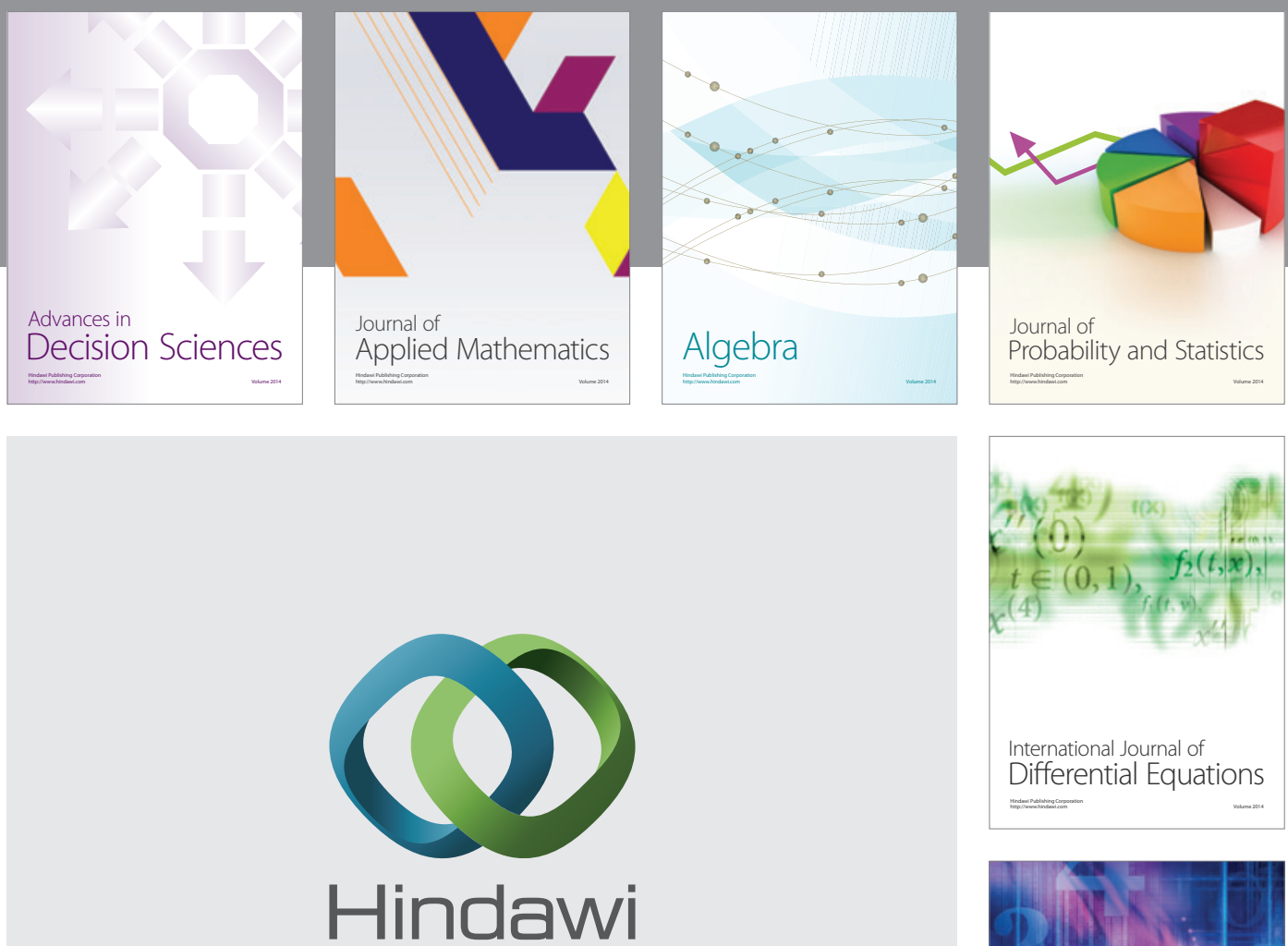

Submit your manuscripts at http://www.hindawi.com
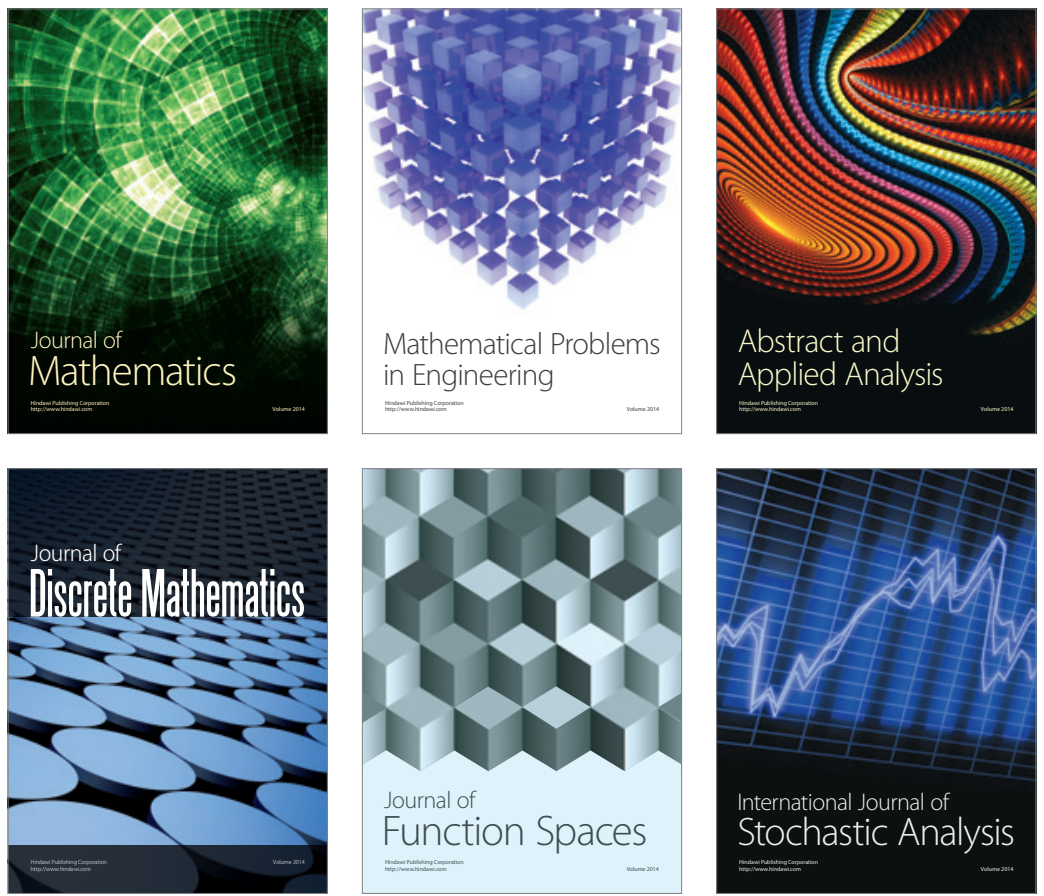

Journal of

Function Spaces

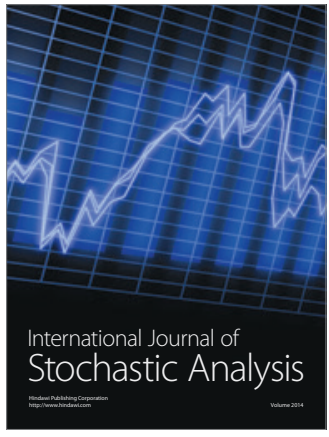

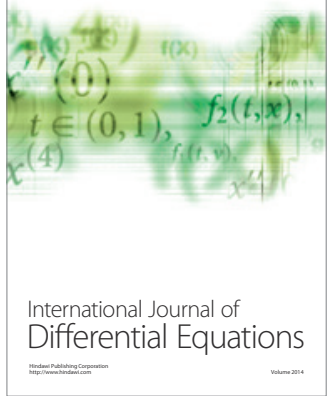
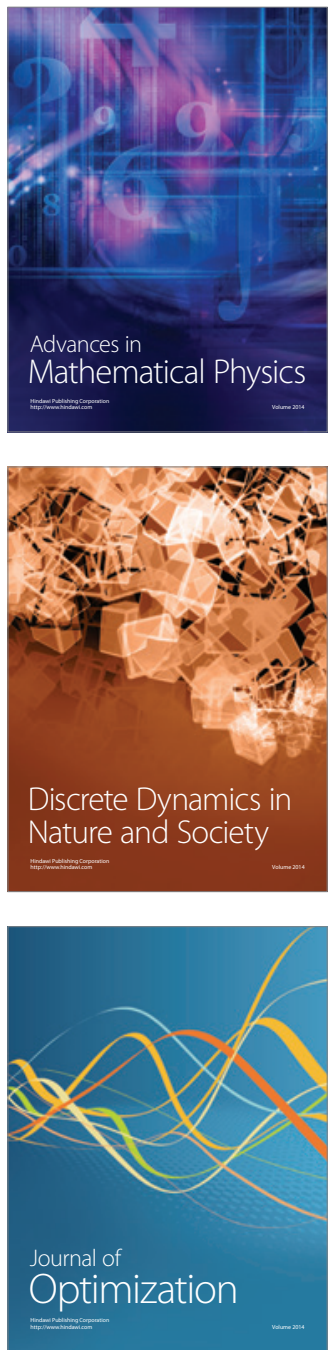